\title{
Research Paper \\ The Effect of Back Massage on Anxiety Components of Mothers With Preterm Infants
}

\author{
Maryam Lali ${ }^{1} \oplus{ }^{*}$ Mina Jouzi ${ }^{1,2} \odot$, Maryam Moghimian ${ }^{1,2}$
}

1. Department of Nursing, School of Nursing and Midwifery, Najafabad Branch, Islamic Azad University, Najafabad, Iran

2. Nursing and Midwifery Sciences Development Research Center, Najafabad Branch, Islamic Azad University, Najafabad, Iran.

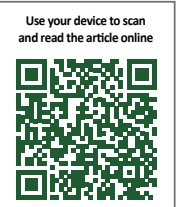

Cittation: Lali M, Jouzi M, Moghimian M. [The Effect of Back Massage on Anxiety Components of Mothers With Preterm Infants (Persian)]. Complementary Medicine Journal. 2020; 9(4):3902-3913. https://doi.org/10.32598/cmja.9.4.933.1

doi'https://doi.org/10.32598/cmja.9.4.933.1

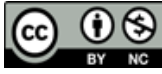

Article Info:

Received: 04 Sep 2019

Accepted: 15 Dec 2019

Available Online: 01 Mar 2020

Keywords:

Premature infant, Mothers, Back massage, Anxiety

\section{A B STRACT}

Objective Upon hospitalization of a premature infant immediately after giving birth, mothers would experience high levels of anxiety. One of the essential non-pharmacological interventions to control the anxiety is massage. The purpose of this study was to investigate the effect of massage on the anxiety of mothers with preterm infants.

Methods In this experimental research, 140 samples were selected based on inclusion criteria using a convenience sampling method and then put in two groups of back massage and one control group. The tools for collecting the data were demographic questionnaire and Lehrer \& Woolfolk's somatic, cognitive, and behavioral anxiety inventory. The data were analyzed using descriptive (Mean $\pm \mathrm{SD}$ ) and inferential statistics tests (Chi-square, independent t-test, paired t-test, Fisher, and covariance analysis) by SPSS V. 13. Results The mean scores of somatic, cognitive, and behavioral anxiety components showed significant differences before and after the intervention in the massage group $(P<0.05)$. There was also a significant difference between the mean anxiety after the intervention $(P<0.05)$.

Conclusion The obtained results indicate that the back massage method is effective in reducing the components of somatic, cognitive, and behavioral anxiety. Hence, this method may be utilized to reduce the anxiety of mothers with premature infants.

\section{Extended Abstract}

\section{Introduction}

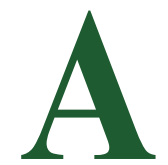

Preterm infant is a baby born before the 37 th week of pregnancy $[1,2]$. Mothers experience a high level of anxiety upon hospitalization of their preterm infant immediately after birth [15]. Anxiety control methods are pharmacological and non-pharmacological (complementary) [17]. Massage is one of the most important non-pharmacological interventions to control anxiety [19]. One of the massage techniques is Swedish massage therapy, which was used to reduce anxiety [22]. The anxiolytic effects of massage have been confirmed in a variety of cases, including primiparous women, post-operative cardiac surgery patients, and patients with heart failure [24-26]. Since the effect of massage on reducing anxiety of mothers with preterm infants has not been investigated so far, this study aimed to investigate this issue.

\section{Materials and Method}

The study was a clinical trial that included mothers with preterm infants hospitalized in Al-Zahra Hospital, and the research environment was the mothers' room. 105 samples

\section{* Corresponding Author:}

Mina Jouzi. PhD.

Address: Department of Nursing, School of Nursing and Midwifery, Najafabad Branch, Islamic Azad University, Najafabad, Iran.

Tel: +98 (31) 42292208

E-mail:minajouzi@pnu.iaun.ac.ir 
were selected by simple sampling method based on inclusion criteria, and then according to the first letter of the mother's surname and after obtaining informed consent from them, they were randomly divided into massage group $(n=35)$ and control group $(n=70)$. The data collection tool consisted of two parts: the first part was demographic characteristics of the research unit and the second part related to the Lehrer Woolfolk physical, cognitive and behavioral anxiety questionnaire. This questionnaire was completed before and after the intervention by questioning the research units of the groups. Back massage was performed using Swedish surface massage techniques for 20 minutes. The control group did not receive any intervention. At the end of the study, the control group received training videos. Data were analyzed using SPSS V. 13 software.

\section{Results}

In this study, 105 mothers with hospitalized preterm infants were divided into two groups of massage $(n=35)$ and control $(n=70)$. Based on the results, demographic data of the mothers in terms of their age, gestational age, and duration of their infant hospital stay did not show any significant difference $(\mathrm{P}=0.05)$ using Chi-square and Fisher's exact tests. Using Chi-square test, the results of the study on the level of education and occupation of mothers with preterm infants did not show any significant difference $(\mathrm{P}>0.05)$ (Table 1$)$. Demographic characteristics of research units were confirmed by homogeneity of groups. The results of dependent t-test showed that the mean of physical, cognitive and behavioral anxiety of mothers with preterm infants did not differ significantly in the intervention group before intervention $(\mathrm{P}>0.05)$.

The mean of physical, cognitive and behavioral anxiety of mothers with preterm infants were significantly different after intervention in the intervention group $(\mathrm{P}<0.05)$. Results of dependent $\mathrm{t}$-test showed that the mean of physical, cognitive and behavioral anxiety of mothers with preterm infants were significantly different $(\mathrm{P}<0.05)$ before and after intervention $(\mathrm{P}<0.05)$ (Table 2$)$, and in control group there was no significant difference $(\mathrm{P}>0.05)$. Statistical test results showed that the mean of physical, cognitive and behavioral anxiety of mothers with preterm infants before and after intervention in massage and control group were significantly different $(\mathrm{P}<0.05)$ (Table 2).

\section{Discussion}

Since the present research was the first study to investigate the effects of back massage on the anxiety components of mothers with preterm infants, the discussion was conducted using studies close to the subject of the research. The results showed that back massage can be effective in reducing anxiety components (physical, cognitive and behavioral). Similarly, the study by Boitor et al. showed that massage is effective in reducing pain and anxiety in patients after

Table 1. Comparison of the frequency distribution of education and occupation of the units under study in the intervention and control groups

\begin{tabular}{|c|c|c|c|c|}
\hline Index & Group & Index Level & Frequency No. (\%) & $\mathbf{P}$ \\
\hline \multirow{12}{*}{ Education level } & \multirow{6}{*}{ intervention } & elementary & $1(0 \% .7)$ & \multirow{12}{*}{0.311} \\
\hline & & intermediate & $6(4 \% .3)$ & \\
\hline & & diploma & $17(12 \% .1)$ & \\
\hline & & associate & $2(1 \% .4)$ & \\
\hline & & bachelor & $6(4 \% .3)$ & \\
\hline & & $\mathrm{MA} / \mathrm{MS}$ & $3(2 \% .1)$ & \\
\hline & \multirow{6}{*}{ control } & elementary & $4(2 \% .9)$ & \\
\hline & & intermediate & $3(2 \% .1)$ & \\
\hline & & diploma & $30(21 \% .4)$ & \\
\hline & & associate & $7(5 \%)$ & \\
\hline & & bachelor & $24(17 \% .1)$ & \\
\hline & & $\mathrm{MA} / \mathrm{MS}$ & $2(1 \% .4)$ & \\
\hline \multirow{4}{*}{ Occupation } & \multirow{2}{*}{ intervention } & housewife & $32(2 \% .9)$ & \multirow{4}{*}{0.561} \\
\hline & & employed & $3(2 \% .1)$ & \\
\hline & \multirow{2}{*}{ control } & housewife & $60(42 \% .9)$ & \\
\hline & & employed & $10(7 \% .1)$ & \\
\hline
\end{tabular}


Table 2. Comparison of mean anxiety components of mothers with preterm infants before and after intervention in massage and control groups

\begin{tabular}{|c|c|c|c|c|}
\hline Group & Variable & Time & Mean \pm SD & $\mathbf{P}$ \\
\hline \multirow{6}{*}{ Massage } & \multirow{2}{*}{ Physical components } & Before intervention & $23.88 \pm 14.98$ & \multirow{2}{*}{0.001} \\
\hline & & After intervention & $12.17 \pm 10.18$ & \\
\hline & \multirow{2}{*}{ Cognitive components } & Before intervention & $35.97 \pm 14.13$ & \multirow{2}{*}{0.001} \\
\hline & & After intervention & $21.88 \pm 12.93$ & \\
\hline & \multirow{2}{*}{ Behavioral components } & Before intervention & $17.71 \pm 10.89$ & \multirow{2}{*}{0.001} \\
\hline & & After intervention & $12.02 \pm 8.09$ & \\
\hline \multirow{6}{*}{ Control } & \multirow{2}{*}{ Physical components } & Before intervention & $19.02 \pm 9.03$ & \multirow{2}{*}{0.217} \\
\hline & & After intervention & $18.74 \pm 9$ & \\
\hline & \multirow{2}{*}{ Cognitive components } & Before intervention & $30.52 \pm 12.84$ & \multirow{2}{*}{0.265} \\
\hline & & After intervention & $29.94 \pm 12.93$ & \\
\hline & \multirow{2}{*}{ Behavioral components } & Before intervention & $18.07 \pm 9.69$ & \multirow{2}{*}{0.056} \\
\hline & & After intervention & $17.68 \pm 9.87$ & \\
\hline
\end{tabular}

cardiac surgery [25]. In the study of Atashi et al. and also in the study of Mohammadpour et al., the effect of Slow Stroke Back Massage (SSBM) on reducing anxiety in patients with stroke and patients undergoing cataract surgery has been mentioned $[28,29]$. The reasons for the present study's concordance with the aforementioned studies include the synchrony of the intervention and the numerical similarity of the samples. Anxiety is an unpleasant feeling that is often accompanied by physical, cognitive, and behavioral symptoms [21]. Massage affects the parasympathetic nerve and can relieve pain, as well as can reduce the effects of heart rate, blood pressure, metabolism, respiratory rate, and anxiety [19]. The results of some studies have shown that massage cannot reduce anxiety, which indicates a discrepancy between the findings of the present research and those studies. For example, the study by Gholami Motlagh et al. suggests that Swedish massage has no effect on anxiety in healthy women [30]. The reason for this discrepancy may be due to the type of samples in the study.

Also, a study by Razmjo et al. showed that foot massage reduced pain severity after cesarean section but did not affect anxiety [31]. This discrepancy may be due to some cultural differences between the study population as well as the nature of the surgeries, the number of sessions, and the duration of the intervention.

\section{Ethical Considerations}

\section{Compliance with ethical guidelines}

All ethical principles were considered in this article. The participants were informed about the purpose of the re- search and its implementation stages; they were also assured about the confidentiality of their information; Moreover, They were allowed to leave the study whenever they wish. This project was registered (Clinical trial Code: IRCT 20190 9120445N1) and ID: IR.IUA.NAJAFABAD. REC1398.054.

Funding

This paper extracted from the master thesis of the first author, Department of Nursing, School of Nursing and Midwifery, Najafabad Branch, Islamic Azad University.

\section{Authors' contributions}

All authors of the article were aware of the submission, completed the commitment form, and participated in all stages.

\section{Conflicts of interest}

The authors did not declare a conflict of interest.

\section{Acknowledgements}

We wish to express our special gratitude to the subjects of the present study and the Vice-Chancellor for Research and Technology of the University as well as to the officials of the Faculty of Nursing and Midwifery of the Islamic Azad University of Najaf Abad Branch who made this research possible. 


\title{
بررسى تأثير ماسار يشت بر مؤلفههاى اضطراب در مادران داراى نوزاد نارس بسترى
}

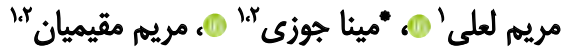

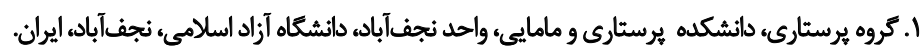

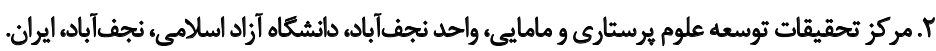

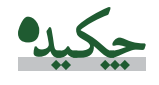

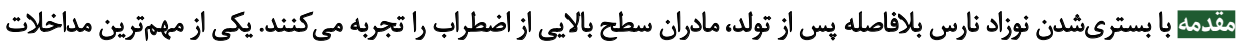

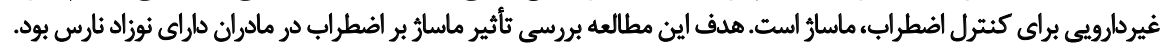

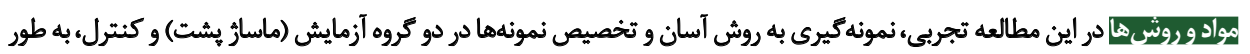

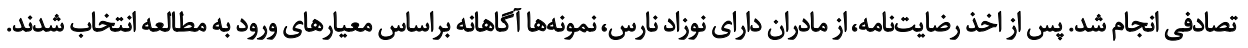

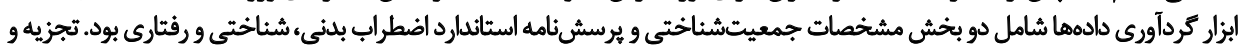

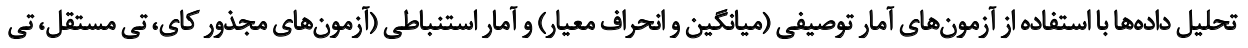

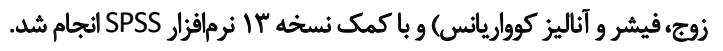

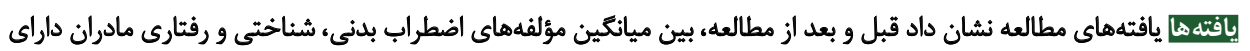

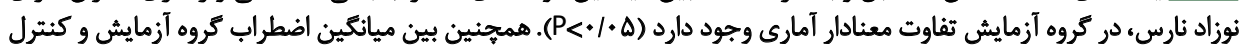

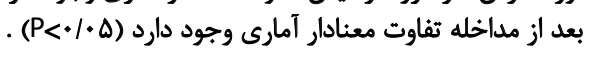

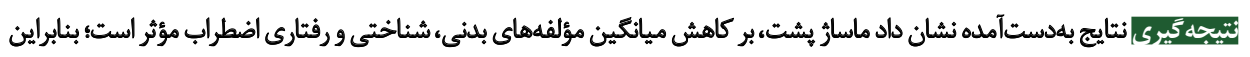

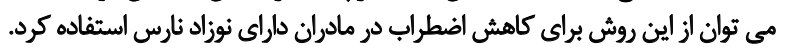

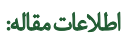 \\ تاريخ دريافت: با شهريور

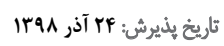

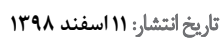

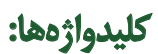
نوزاد نارس، مادر، ماسار

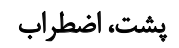

شود. در بخش مراقبتهاى ويرهه مواردى شامل محيط فيزيكى،

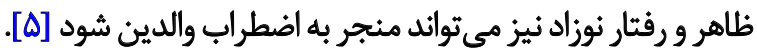

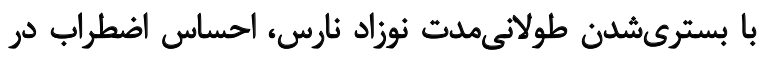

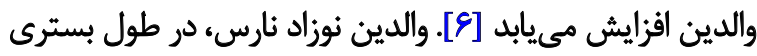

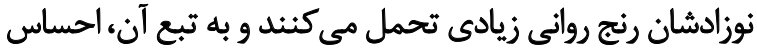

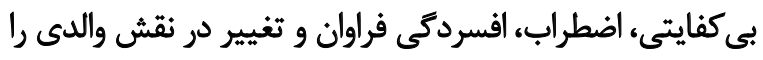

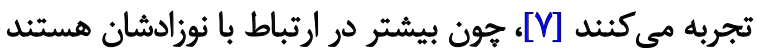

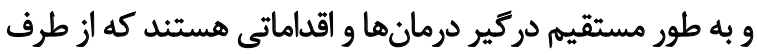

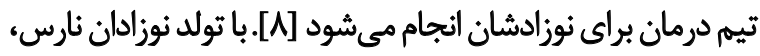

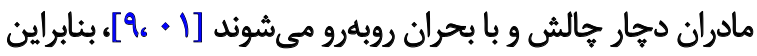

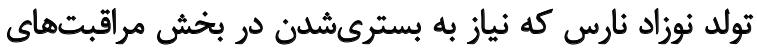

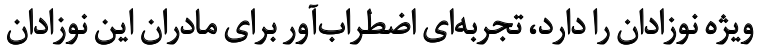

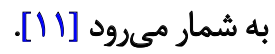

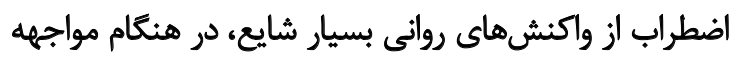

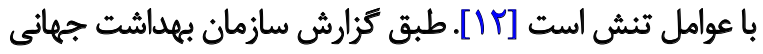

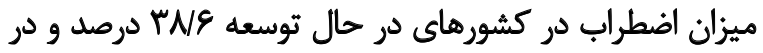

dole

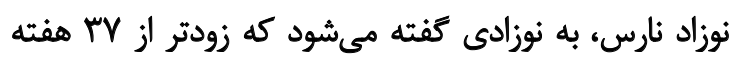

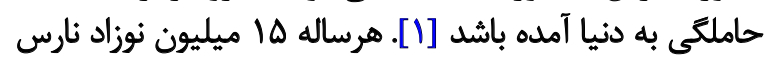

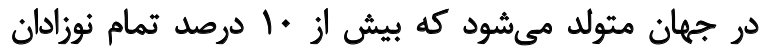

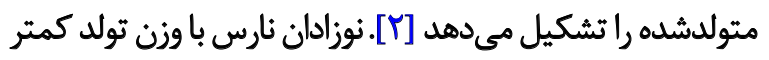

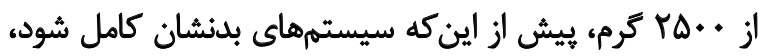

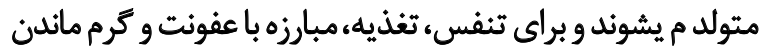

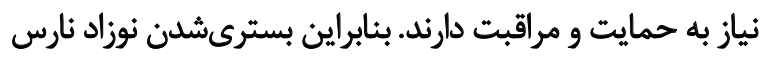

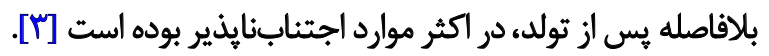

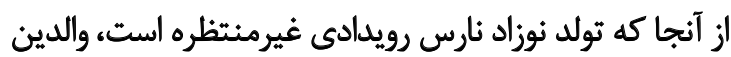

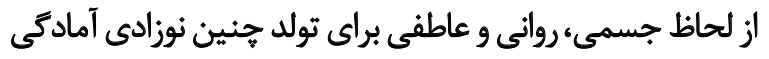

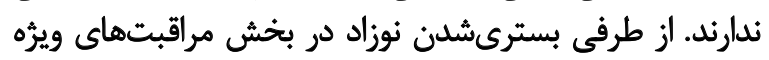

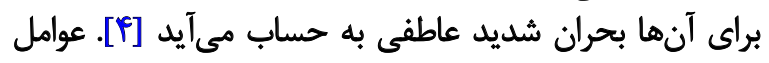

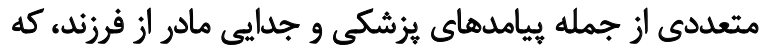

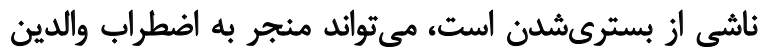


رضايت نامه آكاهانه از مادران نوزادان نارس، اقدام به نمونه ميرى

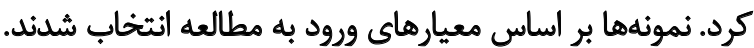
حجم نمونه با فرمول شماره إ محاسبه شد.

\section{1. $n=\left(Z_{1}+a_{2}+Z_{1-\beta}\right)^{2}\left(\sigma_{1}^{2}+\sigma_{2}^{2}\right)$}

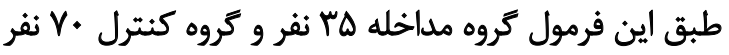

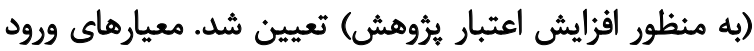

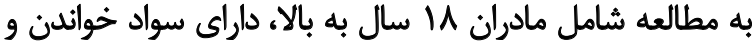

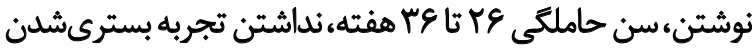

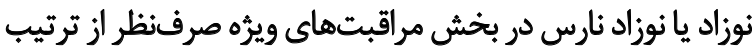

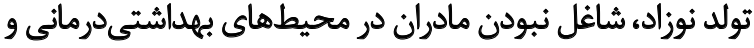

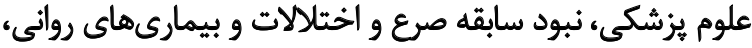

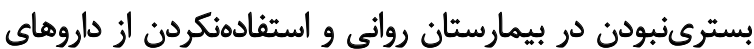

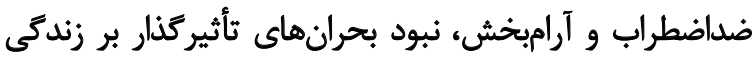

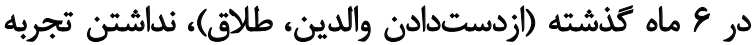

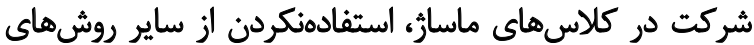

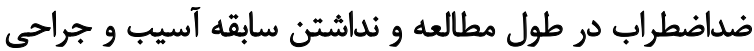

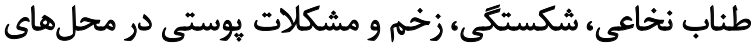

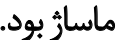

معيارهاى خروج شامل فوت، انتقال و ترخيص نوزاد يا نبود

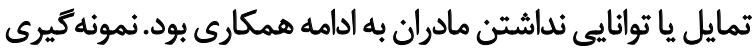

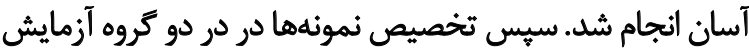

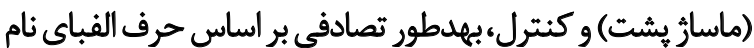

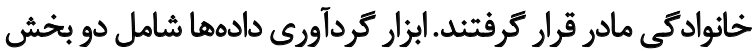

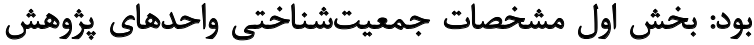

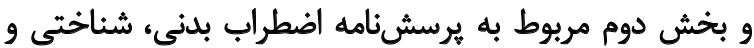

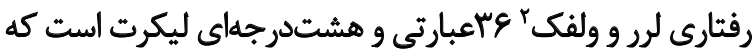

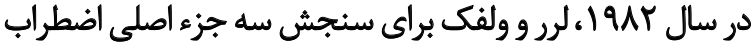

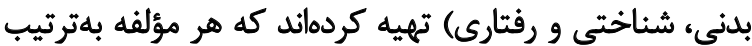

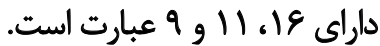

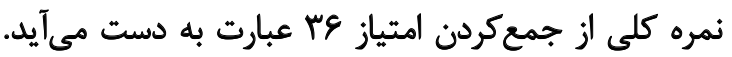

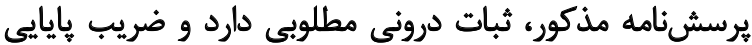

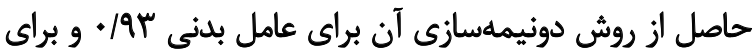

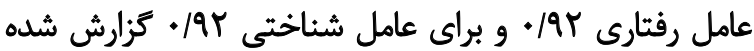

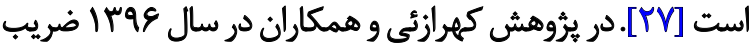

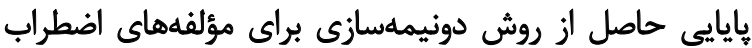

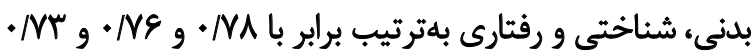

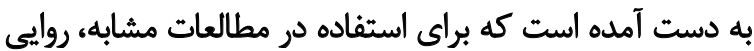

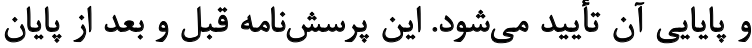

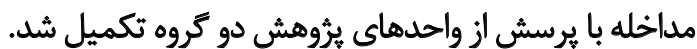

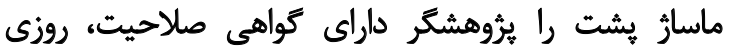

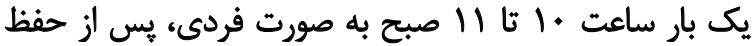

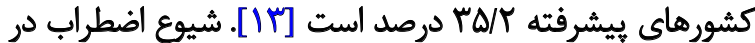

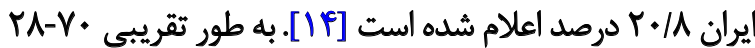

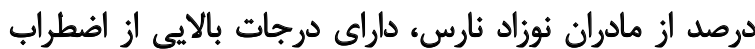

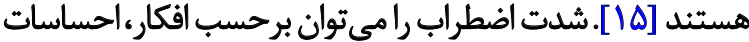

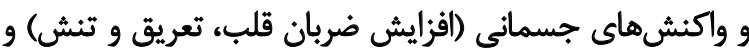

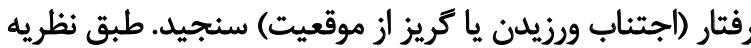

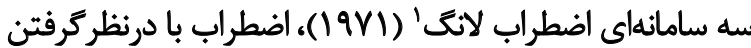

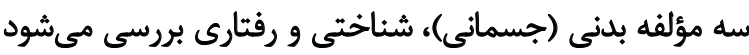

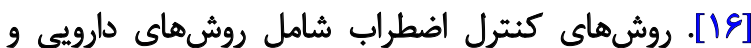
غيردارويى (مكمل) است [IV [IV] در دهاهاى اخير در جهان توجه ويرٔهاى به تركيب درمانهاي

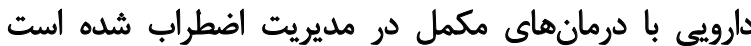

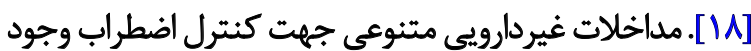

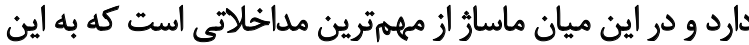

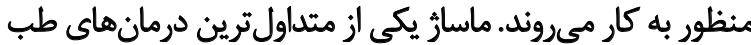

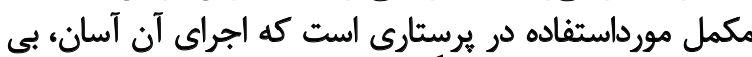

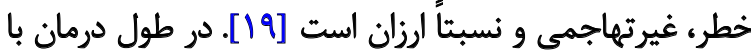

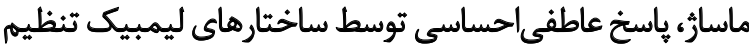

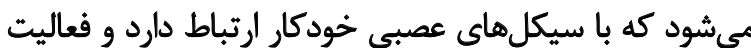

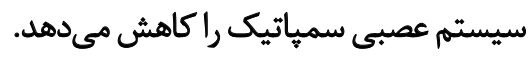

كاهش فعاليت سيستم عصبى سمياتيك مي تواند منجر به

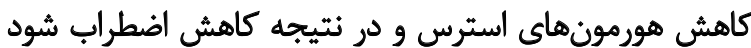

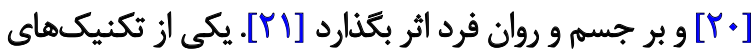

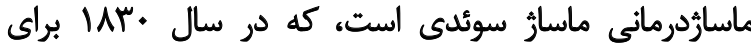

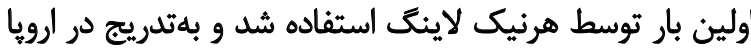

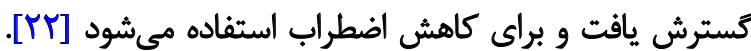

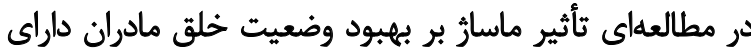

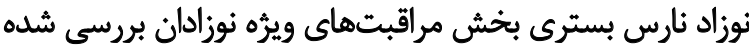

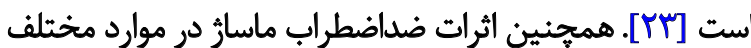

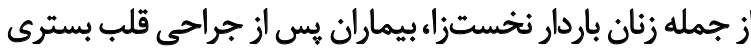

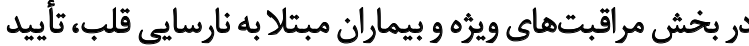

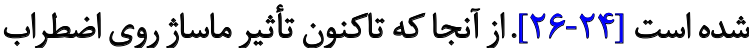

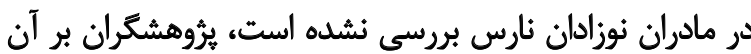

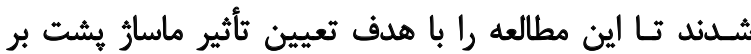
مؤلفههاى اضطراب در مادران داراى نوزاد نارس انجام دين دهئد

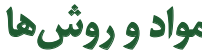

اين برؤوهش تجربى، يس از اخذ مجوز از كميته اخلاق و كد كار

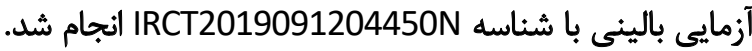

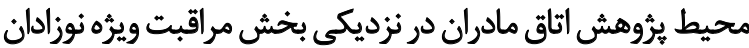

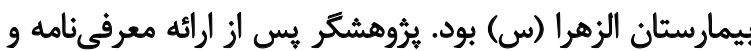

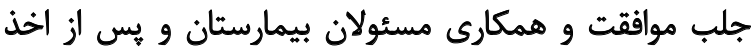


داراى نوزاد نارس از نظر سن، سن حاملكى، مدت بسترى نوزاد،

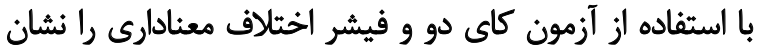

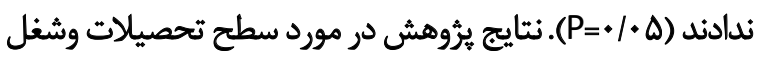

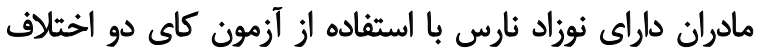

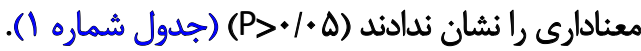

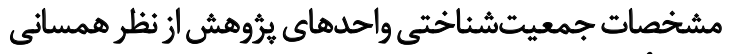

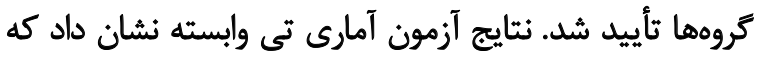

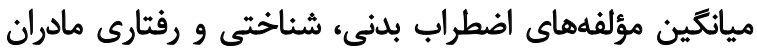

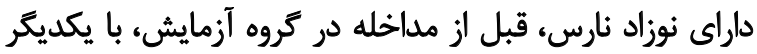

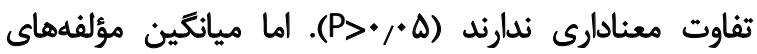

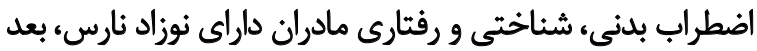

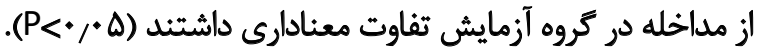

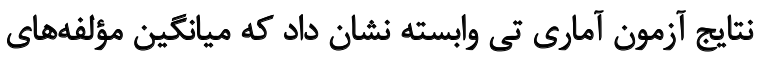

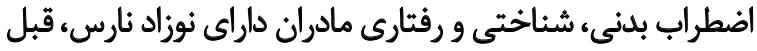

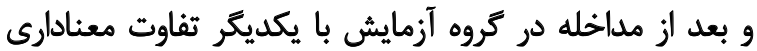

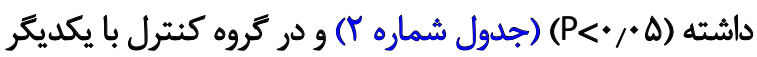

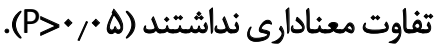

نتايج آزمون آمارى نشان داد كه ميانكين مؤلفههاى اضطراب بدنى،

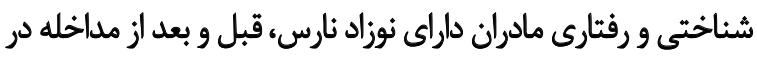

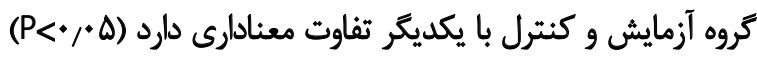
(جدول شماره ب و تصوير شماره ا ).
حريم خصوصى مادران و قراردادن آنها در وضعيتى راحت با بادي

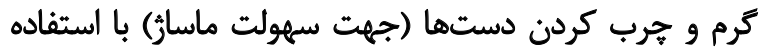

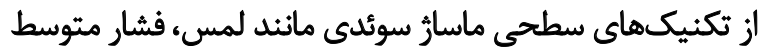

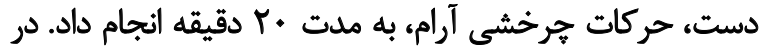

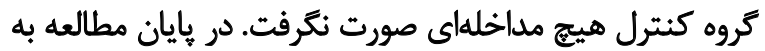

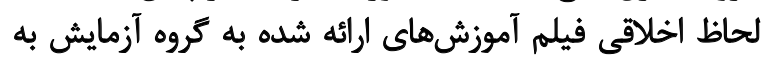

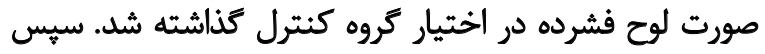
دادههاى جمعآورىشده با استفاده از آزمونهاى آنيت آمار توصيفى

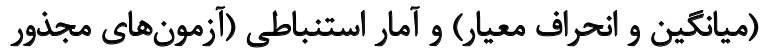

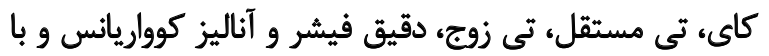

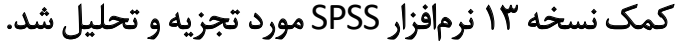

يأقتهها

در اين يُروهش، ه • إدادر داراى نوزاد نارس بسترى شركت

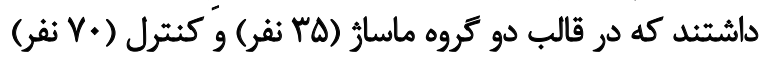

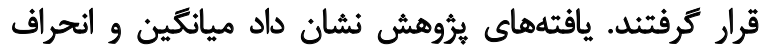

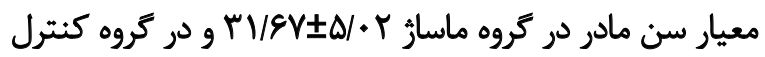

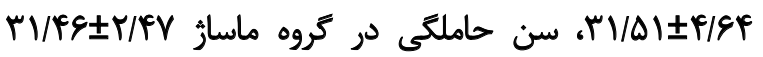
و كروه كنترل

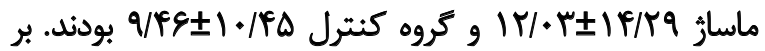

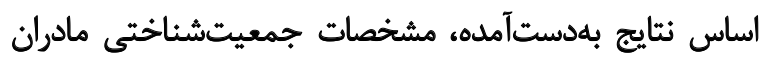

جدول ا. مقايسه توزيع فراوانى تحصيلات و شغل واحدهاى مورد برؤوهش در كروه آزمايش و كثترل

\begin{tabular}{|c|c|c|c|c|}
\hline$P$ & (درصد) فراوانى & سطح شاخص & كروه & شاخص \\
\hline \multirow{12}{*}{. } & $1(\cdot N)$ & ابتدايى & \multirow{6}{*}{ مداخله } & \multirow{12}{*}{ سطح تحصيلات } \\
\hline & $q(\% / \pi)$ & راهنمايى & & \\
\hline & $\operatorname{IV}(I T / 1)$ & دييلم & & \\
\hline & $r(V / r)$ & فوق دييلم & & \\
\hline & $\varepsilon(\kappa / \mu)$ & ليسائس & & \\
\hline & $\Gamma(T / l)$ & فوق ليسانس & & \\
\hline & $F(T / Q)$ & ابتدايى & \multirow{6}{*}{ كنترل } & \\
\hline & $r(r / U)$ & راهنمايى & & \\
\hline & $r \cdot(Y / / P)$ & دييلم & & \\
\hline & $V(\Delta)$ & فوق دييلم & & \\
\hline & $\pi f(I V /)$ & ليسائس & & \\
\hline & $r(1 / F)$ & فوق ليسائس & & \\
\hline \multirow{4}{*}{.$|\Delta S|$} & $r(T / q)$ & خانه دار & \multirow{2}{*}{ ملاخله } & \multirow{4}{*}{ شغل } \\
\hline & $r(Y /)$ & شاغل & & \\
\hline & $e \cdot(f T / q)$ & خانه دار & \multirow{2}{*}{ كتترل } & \\
\hline & $1 \cdot(V / I)$ & شاغل & & \\
\hline
\end{tabular}

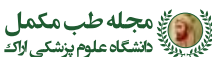


جدول r. مقايسه ميانعين مؤلفه هاى اضطراب مادران داراى نوزاد نارس، قبل و بعد از مداخله در كروه ماسارٌ

\begin{tabular}{|c|c|c|c|}
\hline $\mathbf{P}$ & ميانغين+انحراف معيار & زمان & متغير \\
\hline$\%$ Af & $\mid Q \cdot r \pm q / r$ & قبل از مداخله & \multirow[b]{2}{*}{ مؤلفه هاي بدني } \\
\hline $.1 .0 Y$ & $W V^{e} \pm q$ & بعد از مداخله & \\
\hline .1 .8 & $r \cdot / \Delta r \pm I r / A r$ & قبل از مداخله & \multirow{2}{*}{ مؤلفه هاي شُناختى } \\
\hline $.0 r$ & rqqf \pm Ir/qr & بعد از مداخله & \\
\hline - $/ A E \Delta$ & $W \cdot V \pm q / e q$ & قبل از مداخله & \multirow{2}{*}{ مؤلفه هاى رفتارى } \\
\hline $.1+4$ & IV/\&A \pm V/AV & بعد از مداخله & \\
\hline
\end{tabular}

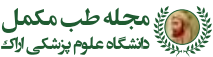

جدول "ا. مقايسه ميانكين مؤلفه هاي اضطراب مادران داراى نوزاد نارس، قبل و بعد از مداخله دركروه ماسار و كثترل

\begin{tabular}{|c|c|c|c|c|}
\hline $\mathbf{P}$ & ميانغين ثانحراف معيار & زمان & متغير & كروه \\
\hline $.1 .+1$ & $\begin{array}{l}r r / M \pm I F / M \\
I r / M V \pm 1+/ M\end{array}$ & قبل از مداخله & مؤلفه هاى بلدنى & \\
\hline$+1+\infty 1$ & $\begin{array}{l}r \Delta / q \vee \pm \mid r / / \mu \\
r V / M \pm \mid r / q \mu\end{array}$ & قبل ازز مداخله & مؤلفه هاى شناختى & ماسائز \\
\hline$+1+.1$ & $\begin{array}{l}|V / N| \pm 1 \cdot / 19 \\
\mid r / \cdot r \pm N \cdot q\end{array}$ & قبل ازز مداخله & مؤلفه هاى رفتارى & \\
\hline.$/$ TIV & $\begin{array}{l}\mid V \cdot r \pm q / r \\
W H V^{2} \pm q\end{array}$ & قبل از معاخله & مؤلفه هاي بدنى & \\
\hline . /TEO & $\begin{array}{l}r \cdot / \Delta Y \pm I r / A f \\
\text { ra/ar } \pm \text { Ir/ar }\end{array}$ & قبل از مداخله & مؤلفه هاى شناختي & كثترل \\
\hline.$/ . \Delta P$ & $\begin{array}{l}W \cdot V \pm q / \varepsilon q \\
W V / F A \pm q / A V\end{array}$ & قبل از مداخله & مؤلفه هاى رقثتارى & \\
\hline
\end{tabular}

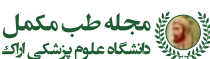

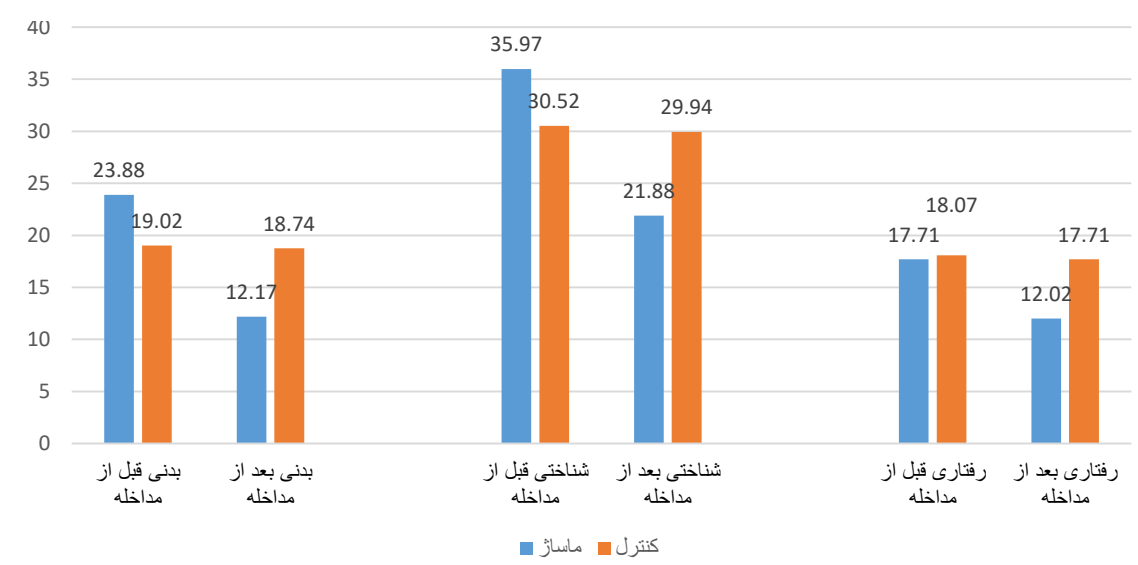


ممكن است برخي ثفاوتهاي فرهنكي بين جمعيت مورد

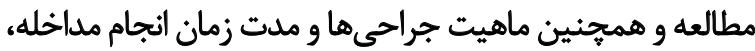

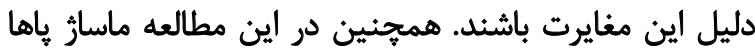

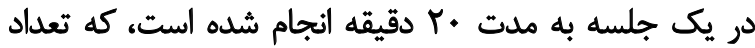

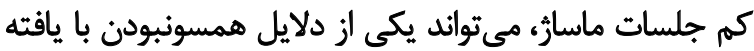
يروهش حاضر باشد.

\section{تتيجه}

در مجموع مىتوان جنين نتيجه كيرى نمود كه ماسازٌ يشت مإثت

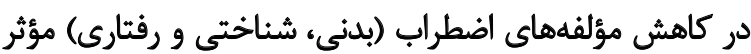

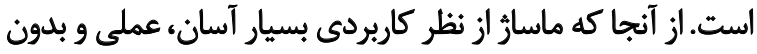

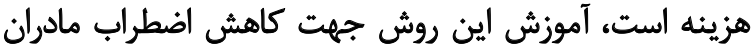

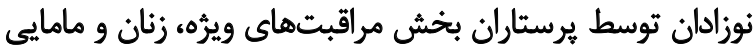

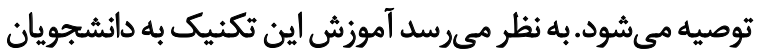

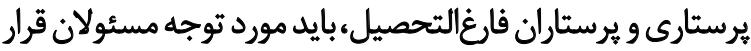
كيرد.

محدوديتهاي مطالعه شامل وجود تفاوتهايي از نظر فرهنكي،

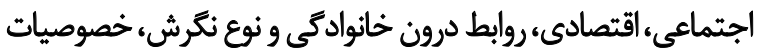

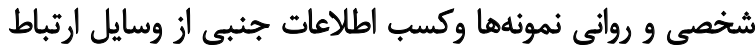

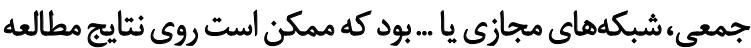

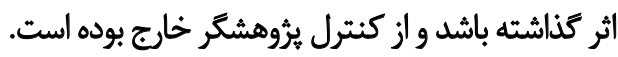

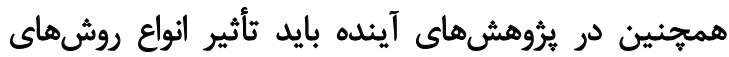

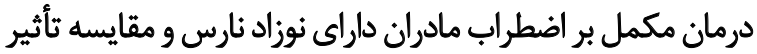

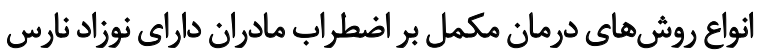

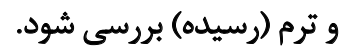

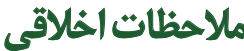 \\ ييروى ازٔ اصول اخلاق ئروهش}

همه اصول اخلاقى در اين مقاله رعايت شده است.

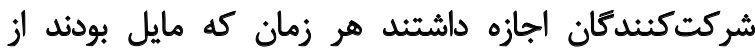

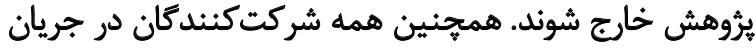

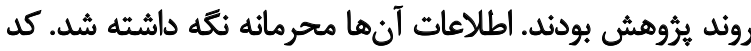
كارآزمايى بالينى IR.IAU.NAJAFABAD.REC.1398.054 بود.

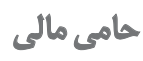

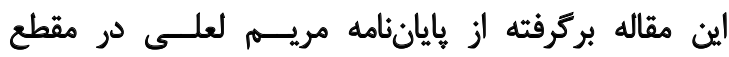

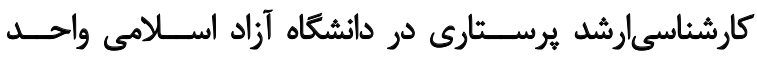

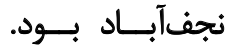

$$
\text { مشار كت ثويسند كان }
$$

نويسندكان به يك اندازه در نكارش مقاله مشاركت داشتهاند.
با توجه به اينكه يُؤوهش حاضر، اولين يروهشى است كه

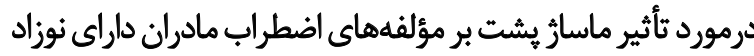

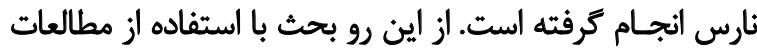

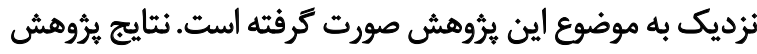

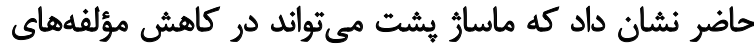

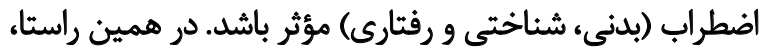

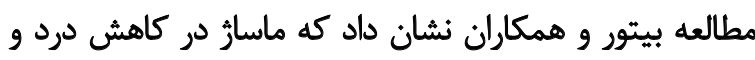

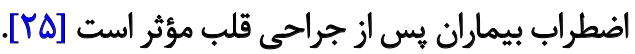

مطالعه آتشى و همكاران نيز به مؤثر بودن ماسار استروى

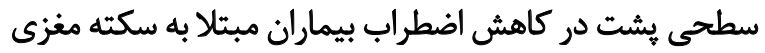

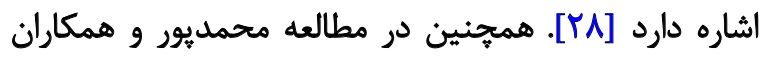

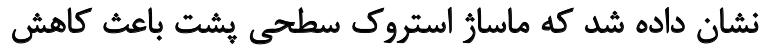

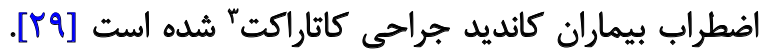

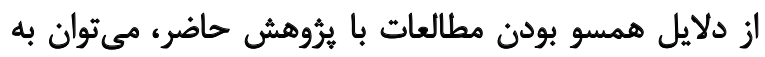

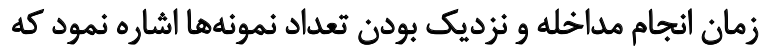

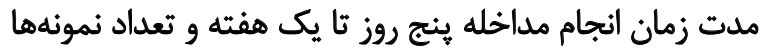

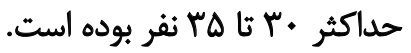

اضطراب احساسى ناخوشايند است كـه در افراد به واسطه

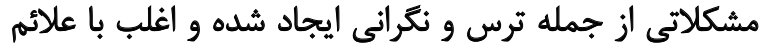

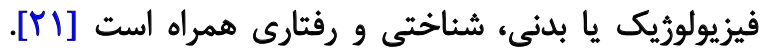

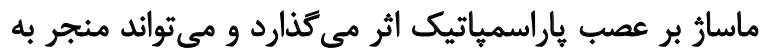

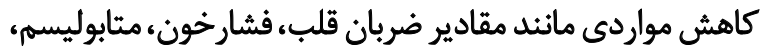

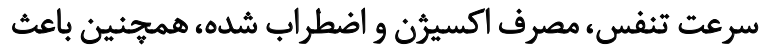

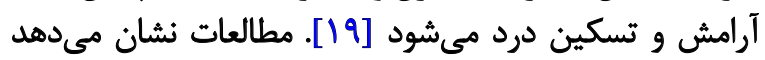

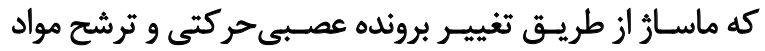

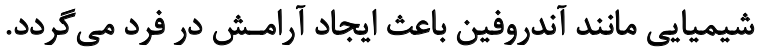

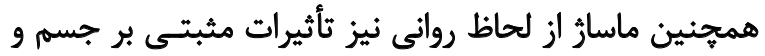

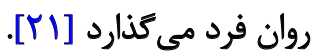

نتايج مطالعاتى نيز نشان دادهاند كه ماسار نمى تواند ميزان إنان

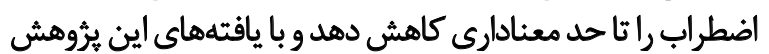

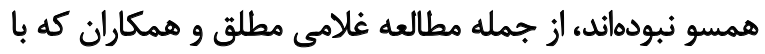
انجام دو روش ماساز سوئدى بر زنان سالم، نشان دادئ دادئد ميانكين

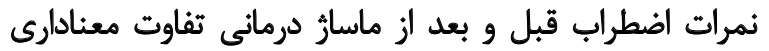

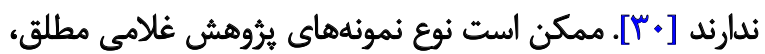

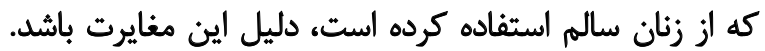

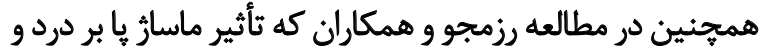

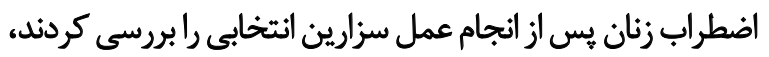

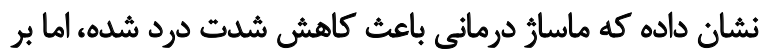
اضطراب مؤثر نبوده است [آس] داني 


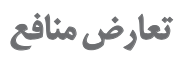

بنابر اظهار نويسندكان اين مقاله تعارض منافع نداشته است.

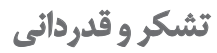

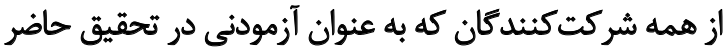

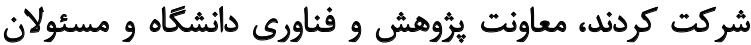

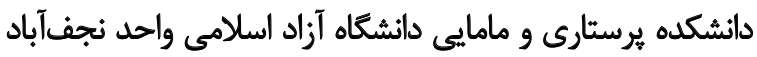

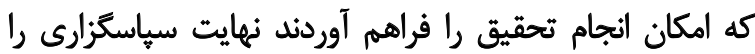
داريم. 


\section{References}

[1] Martin RJ, Fanaroff AA, Walsh MC. Fanaroff and Martin's neonatal-perinatal medicine: Diseases of the fetus and infant. St. Louis: Mosby; 2011. [DOI:10.1016/B978-0-323-06545-0.00062-5]

[2] Blencowe H, Cousens S, Chou D, Oestergaard M, Say L, Moller $A B$, et al. Born too soon: The global epidemiology of 15 million preterm births. Reproductive Health. 2013; 10(Suppl 1):S2 [DOI:10.1186/1742-4755-10-S1-S2] [PMID] [PMCID]

[3] Hosseini SS, Baniasadi H, Pouraboli B. [Stressors of parents of hospitalized preterm infants: A study in neonatal intensive care unit of afzalipour Hospital, Kerman, Iran (Persian)]. Health and Development Journal. 2015; 4(4):337-48. http://jhad.kmu.ac.ir/ article-1-119-en.html

[4] Hollywood M, Hollywood E. The lived experiences of fathers of a premature baby on a neonatal intensive care unit. Journal of Neonatal Nursing. 2011; 17(1):32-40. [DOI:10.1016/j. jnn.2010.07.015]

[5] Blanch D'Souza SR, Karkada S, Lewis LE, Mayya Sh, Guddattu V. Relationship between stress, coping and nursing support of parents of preterm infants admitted to tertiary level neonatal intensive care units of Karnataka, India: A cross-sectional survey. Journal of Neonatal Nursing. 2009; 15(5):152-8. [DOI:10.1016/j. jnn.2009.07.003]

[6] Smith M. Good parenting: Making a difference. Early Human Development 2010; 86(11):689-93. [DOI:10.1016/j.earlhumdev.2010.08.011] [PMID]

[7] Bastani F, Ali Abadi T, Haghani H. [The effectiveness of participatory care program in neonatal intensive care unit on state anxiety of mothers of preterm newborns (Persian)]. Journal of Babol University of Medical Sciences. 2012; 14(3):59-65. https://www. researchgate.net/publication/305357399

[8] Mackley AB, Locke RG, Spear ML, Joseph R. Forgotten parent: NICU paternal emotional response. Advances in Neonatal Care. 2010; 10(4):200-3. [DOI:10.1097/ANC.0b013e3181e946f0] [PMID]

[9] Spielman V, Taubman-Ben-Ari O. Parental self-efficacy and stress-related growth in the transition to parenthood: A comparison between parents of pre-and full-term babies. Health \& Social Work. 2009; 34(3):201-12. [DOI:10.1093/hsw/34.3.201] [PMID]

[10] Cockcroft S. How can family centred care be improved to meet the needs of parents with a premature baby in neonatal intensive care? Journal of Neonatal Nursing. 2012; 18(3):105-10. [DOI:10.1016/j.jnn.2011.07.008]

[11] Lee SY, Hsu HC. Stress and health-related well-being among mothers with a low birth weight infant: The role of sleep. Social Science \& Medicine. 2012; 74(7):958-65. [DOI:10.1016/j.socscimed.2011.12.030] [PMID] [PMCID]

[12] Kahrazei F, Hashemzehi S, Rigi Kooteh B. [The effect of cognitive behavioral therapy on components of anxiety (physical, behavioral and cognitive) in patients with thalassemia major (Persian)]. Middle Eastern Journal of Disability Studies. 2017; 7:1-7. http:// jdisabilstud.org/article-1-792-en.html

[13] Modara F, Sarokhani D, Valizadeh R, Sarokhani M. [The average review score of anxiety in Iran: Systematic review and metaanalysis (Persian)]. Journal of Ilam University of Medical Sciences. 2017; 25(2):181-206. [DOI:10.29252/sjimu.25.2.181]
[14] Fallahzadeh H, Ghafarikia M, Keyghobady N, Saadati H. [Comparison of depression and anxiety in employed women with housewives women in Shiraz City in 2012 (Persian)]. The Journal of Toloo-e-behdasht. 2014; 13(1):115-23. http://tbj.ssu.ac.ir/ article-1-1599-en.html

[15] Jafari Mianaei S, Alaee Karahroudy F, Rasuli M, Zayeri F. [Effectiveness of "Parent Empowerment" program on anxiety and stress in mothers who have preterm infants hospitalized in NICUs (Persian)]. Payesh. 2012; 11(2):253-8. http://payeshjournal.ir/article-1-474-fa. html

[16] Hosseinpour MR, Samiei L, Alizadeh A. [Four systems anxiety assessment in hemodialysis patients (Persian)]. Beyhagh. 2015; 20(1):3341. https://www.sid.ir/fa/journal/ViewPaper.aspx?ID=281752

[17] Torabi M, Salavati M, Ghahri Sarabi AR, Pooresmaeel Z, Akbarian Baghban AR. [Effect of foot reflexology massage and benson relaxation techniques on anxiety and physiological indexes of patients undergoing coronary heart angiography (Persian)]. Avicenna Journal of Nursing and Midwifery Care. 2012; 20(1):63-73. http://nmj.umsha. ac.ir/article-1-1108-en.html

[18] Wong LY, Toh MPHS, Kong KH. Barriers to patient referral for complementary and Alternative medicines and its implications on interventions. Complementary Therapies in Medicine. 2010; 18(3-4):13542. [DOI:10.1016/j.ctim.2010.05.034] [PMID]

[19] Bazrafshan MR, Ghorbani Z. [The effect of Slow Stroke Back Massages on anxiety among primigravid women (Persian)]. Journal of Hayat. 2010; 16(1):34-40. http://hayat.tums.ac.ir/article-1-95-fa. htm

[20] Jouzi M. [Assessment of the effect of massage therapy on stroke patients (Persian)]. Medical Science Journal of Islamic Azad University Tehran Medical Branch. 2009; 19(4):256-61. http://tmuj.iautmu. ac.ir/article-1-225-en.html

[21] Babashahi M, Fayaz S, Mardanian Dehkordi L. [Comparing the effect of massage and inhalation aromatherapy on anxiety level of the patients in the preoperative period (Persian)]. Nursing and Midwifery Journal. 2015; 13(4):284-91. http://unmf.umsu.ac.ir/article1-1432-en.htm

[22] Kashaninia Z, Abedinipoor A, Hosainzadeh S, Sajedi F. The effect of swedish massage on glycohemoglobin in children with diabetes mellitus. Iranian Rehabilitation Journal. 2011; 9:16-20. http://irj.uswr. ac.ir/article-1-209-en.pdf

[23] Tirgari B. [The effect of massage by mother on the situation of mood admitted to the NICU of the Afzalipour Hospital of Kerman University of Medical Sciences in 2016 (Persian)] [MSc. thesis]. Kerman: Kerman University of Medical Sciences; 2016. https://fa.irct.ir/ trial/20942? revision=20942

[24] Khojasteh F. [Comparing the effects of massage and guided imagery during pregnancy on anxiety among nulliparous women (Persian)] [MSc. thesis]. Zahedan: Zahedan University of Medical Sciences and Health Services; 2016. https://fa.irct.ir/trial/23267

[25] Madalina Boitor, Géraldine Martorella, Christine Maheu, Andréa Maria Laizner, Céline Gélinas. Effects of Massage in Reducing the Pain and Anxiety of the Cardiac Surgery Critically III-a Randomized ControlledTrial Pain Medicine. 2018;19(12):2556-69. [DOI:10.1093/ $\mathrm{pm} /$ pny055] [PMID]

[26] Chen WL, Liu CJ, Yeh SH, Chiang MC, Fu MY, Hsieh YK. Effect of Back Massage Intervention on Anxiety, Comfort, and Physiologic Responses in Patients with Congestive Heart Failure. The Journal of Alternative and Complementary Medicine. 2013; 19(5):464-470. [DOI:10.1089/acm.2011.0873] 
[27] Corcoran KJ, Fisher J. Measures for clinical practice and research: A sourcebook. Fifth ed. New York: Oxford University Press; 2013.

[28] Atashi V, Mohammadi F, Dalvandi A, Abdellahi I, Shafiei Z. [Effect of slow stroke back massage on anxiety in patients with stroke: A randomized clinical trial (Persian)]. Journal of Clinical Nursing and Midwifery. 2014; 2(4):45-52. http://jcnm.skums.ac.ir/article-1-63-fa. html

[29] Keramati M, Sargolzaei MS, Moghadasi A, Basirinezhad MH, Mohammadpourhodki R. Evaluating the effect of Slow-Stroke Back Massage on the anxiety of candidates for cataract surgery. International Journal of Therapeutic Massage \& Bodywork. 2019; 12(2):12-7. [PMID] [PMCID]

[30] Gholami-Motlagh F, Jouzi M, Soleymani B. Comparing the effects of two Swedish massage techniques on the vital signs and anxiety of healthy women. Iranian Journal of Nursing and Midwifery Research. 2016; 21(4):402-9. [PMID] [PMCID]

[31] Razmjoo N, Hafizi Lotfabadi L, Yousefi F, Esmaeeli H, Azizi H, Lotfalizadeh $\mathrm{M}$. [Effect of foot reflexology on pain and anxiety in women following elective cesarean section (Persian)]. The Iranian Journal of Obstetrics, Gynecology and Infertility. 2012; 15(1):8-16. http:// eprints.mums.ac.ir/4433/ 
This Page Intentionally Left Blank 